\title{
Bất bình đẳng thu nhập theo giới tính và tăng trưởng kinh tế địa phương tại Việt Nam
}

\section{Gender-based income inequality and provincial economic growth in Vietnam}

\author{
Võ Hồng Đức ${ }^{1 *}$, Nguyễn Công Thắng ${ }^{1}$, Hồ Minh Chí ${ }^{1}$, Võ Thế Anh ${ }^{1}$, Phạm Ngọc Thạch ${ }^{1}$ \\ ${ }^{1}$ Trường Đại học Mở Thành Phố Hồ Chí Minh, Việt Nam \\ *Tác giả liên hệ, Email: duc.vhong@ou.edu.vn
}

\section{THÔNG TIN}

DOI: $10.46223 / \mathrm{HCMCOUJS.}$ econ.vi.13.3.505.2018

Ngày nhận: 10/08/2018

Ngày nhận lại: 24/09/2018

Duyệt đăng: 31/10/2018

Tù khóa:

bất bình đẳng thu nhập theo giới, chỉ số Gini, chỉ số Theil, tăng trưởng kinh tế, Việt Nam

Keywords:

gender-based income inequality, Gini index, Theil index, economic growth, Vietnam
TÓM TÁT

Quan hệ giữa bất bình đẳng thu nhập và tăng trưởng kinh tế đã thu hút nhiều sự chú ý của các nhà nghiên cứu kinh tế và cơ quan hoạch định chính sách. Tuy nhiên, bất bình đẳng thu nhập theo giới tính vẫn chưa dành được nhiều sự quan tâm, đặc biệt trong bối cảnh của các quốc gia mới nổi như Việt Nam. Nghiên cứu này được thực hiện để đánh giá và lượng hóa tác động của bất bình đẳng thu nhập theo giới lên tăng trưởng kinh tế địa phương cho 63 Tỉnh/thành trong giai đoạn 2004-2014, thời điểm mới nhất dữ liệu nghiên cứu có được từ cuộc khảo sát của Tổng cục Thống kê. Trong nghiên cứu này, bất bình đẳng thu nhập theo giới tính được đo lường bởi hai chỉ số khác nhau: (i) Chỉ số Gini; và (ii) Chỉ số Theil. Kết quả nghiên cứu chỉ ra rằng trong khi bất bình đẳng thu nhập theo nam giới có tác động và tác động cùng chiều đến tăng trưởng kinh tế, bất bình đẳng thu nhập theo nữ giới lại có tác động ngược chiều đến tăng trưởng kinh tế địa phương tại Việt Nam trong thời gian vừa qua.

\section{ABSTRACT}

The link between income inequality and economic growth has drawn great attention from economists and policy-makers around the globe. However, gender-based income inequality has been largely ignored in current literature, particularly in emerging markets such as Vietnam. This study examines the impact of gender wage inequality on economic growth of 63 provinces in Vietnam during the period of 2004-2014 using existing data from most recent surveys. In this study, income inequality is measured using: (i) Gini Index and (ii) Theil Index. The research findings indicate that male-based income inequality plays a significant role and positively impacts economic growth 
while female-based income inequality negatively affects economic growth in Vietnam.

\section{Giới thiệu}

Trong báo cáo "Sự phân chia trong xã hội loài người: Đối diện với bất bình đẳng ở các nước đang phát triển" (United Nations Development Programme, 2013), bất bình đẳng trong thu nhập được xem là thước đo cho sự phân phối thu nhập giữa các hộ gia đình, giữa các cá nhân trong một nền kinh tế. Trong báo cáo nói trên, Ngân hàng thế giới tập trung phân tích phân phối thu nhập chủ yếu giữa hai đối tượng: Phân phối thu nhập hộ gia đình và Phân phối thu nhập dựa vào chức năng (chức năng sản xuất).

Theo United Nations Development Programme (2013), bất bình đẳng trong thu nhập là vấn đề điển hình được đánh giá trong lý thuyết về quỹ đạo tăng trưởng dài hạn được xem xét cho các quốc gia đang phát triển (được biết đến nhiều nhất là Lewis (1954) và Kuznets (1955). Trong các tiếp cận trên về bất bình đẳng thu nhập, trong giai đoạn mới bắt đầu của tăng trưởng, tăng trưởng và phân phối thu nhập tồn tại như hai mặt phải đánh đổi cho nhau. Ý kiến này được minh chứng qua giả thuyết nổi tiếng về Đường cong chữ U ngược của Kuznets, một khung phân tích tổng quát về bất bình đẳng trong lý thuyết tăng trưởng kinh tế hiện đại giải thích chiều hướng tăng lên đáng kể của thu nhập trung bình của quốc gia (Kuznets, 1955).

Giả thuyết của Kuznets lập luận dựa trên hai giả định: (i) có sự khác biệt rõ ràng trong thu nhập của hai nhóm ngành nông nghiệp - nông thôn và công nghiệp - thành thị, và (ii) bất bình đẳng tăng lên nhiều hơn trong nội ngành của ngành công nghiệp - thành thị so với ngành nông nghiệp - nông thôn. Theo chiều hướng dịch chuyển lao động từ nơi dư thừa lao động ở ngành nông nghiệp sang nơi thiếu hụt ở ngành công nghiệp, sức hút của ngành có bất bình đẳng cao sẽ tăng lên trong khi khoảng cách thu nhập giữa hai nhóm ngành cũng có xu hướng dãn rộng hơn. Như vậy, bất bình đẳng nhìn chung sẽ tăng lên và giữ vững ổn định trong một khoảng thời gian trước khi giảm xuống. Câu hỏi đặt ra là tại sao bất bình thu nhập lại giảm xuống? Với Kuznets, bất bình đẳng thu nhập "sau cùng sẽ giảm dần bởi sự trổi dậy của nhóm dân cư có thu nhập thấp về mặt kinh tế và chính trị sau sự sai lệch về phân phối nguồn lực trong giai đoạn đầu tiên của cuộc cách mạng công nghiệp, sau đó, những người có thu nhập thấp sẽ dần trở thành cư dân thành thị $[\ldots]$ và đó là sự chuyển đổi xã hội, đó là điều cơ bản để phá vỡ bất bình đẳng thu nhập của một quốc gia" (McKinley, 2009).

Nếu như tăng trưởng kinh tế và bất bình đẳng thu nhập là một sự đánh đổi, thì các hàm ý chính sách từ giả thuyết của Kuznets là rất rõ ràng. Chính sách phát triển cần phải xem xét bản thân vấn đề của phát triển cùng với tăng trưởng kinh tế. Các quan ngại về phân phối thu nhập cần được gạt bỏ. Lập luận này nhấn mạnh vào khía cạnh tăng trưởng kinh tế, được biện luận bằng nhiều bằng chứng nghiên cứu cho thấy rằng những nhà tư bản có khuynh hướng tiết kiệm nhiều hơn so với những người công nhân, theo đó thì các chính sách về phân phối lại thu nhập dựa trên lợi nhuận sẽ thúc đẩy tăng trưởng kinh tế (Goodwin, 1967; Kaldor, 1957). Nói theo cách khác, bất bình đẳng thu nhập dường như có ảnh hưởng tích cực hơn là tiêu cực đến tăng trưởng kinh tế. 
Nền kinh tế Việt Nam đã và đang bắt đầu đạt được những thành tựu cực kỳ quan trọng và ấn tượng. Nền kinh tế chúng ta đã và đang bắt đầu hội nhập sâu và rộng với nền kinh tế thế giới. Tăng trưởng kinh tế tại Việt Nam trong 2 thập kỷ vừa qua nhận được sự quan tâm của rất nhiều quốc gia trên thế giới. Tuy nhiên, hố sâu chênh lệch thu nhập cũng tăng dần lên theo thời gian. Báo cáo mới nhất của Oxfam (2017) thể hiện rằng, khoảng 260 người giàu nhất của Việt Nam có thể đưa hơn 3.2 triệu người Việt nam ra khỏi ngưỡng nghèo khó. Thông qua việc lược khảo cơ sở lý thuyết và các nghiên cứu, chúng tôi không tìm thấy bất kỳ nghiên cứu nào được thực hiện nhằm mục đích cung cấp một bằng chứng khoa học định lượng tại Việt Nam cho vấn đề bất bình đẳng về giới và tăng trưởng kinh tế địa phương ở Việt Nam trong giai đoạn vừa qua. Do vậy, nghiên cứu này được thực hiện nhằm cung cấp một lời giải thích thỏa đáng cho vấn đề quan trọng này tại Việt Nam. Kết quả của nghiên cứu sẽ cung cấp một bằng chứng khoa học định lượng cho cơ quan quản lý kinh tế nhà nước tại Việt Nam trong tương lai.

\section{Cơ sở lý thuyết}

Các lý thuyết nền tảng của kinh tế học vĩ mô liên quan đến tăng trưởng kinh tế và bất bình đẳng thu nhập rất nhiều. Mức độ phổ biến của các lý thuyết này vượt ra khỏi phạm vi của nghiên cứu này.

Trên phương diện nghiên cứu định lượng, rất nhiều các nghiên cứu được thực hiện để đánh giá mối quan hệ giữa tăng trưởng kinh tế và rất nhiều các khía cạnh kinh tế học khác. Mối quan hệ giữa tăng trưởng kinh tế và bất bình đẳng thu nhập được thực hiện rất nhiều trên phạm vi toàn thế giới. Một số nghiên cứu tiêu biểu có thể được kể đến bao gồm nghiên cứu của Persson và Tabellini (1994); Lin, Huang, và Yeh (2014); Fawaz, Rahanama và Valcarcel (2014); và Biswas, Chakraborty, và Hai (2017) đã xem xét đến việc đánh giá và lượng hóa tác động từ bất bình đẳng thu nhập đến tăng trưởng kinh tế.

Persson và Tabellini (1994) thực hiện nghiên cứu về tác động của phân phối thu nhập lên mức thu nhập giữa các quốc gia. Tác giả phân tích tác động này dựa vào hai lý thuyết nền tảng là (i) lý thuyết tăng trưởng nội sinh và (ii) lý thuyết chính sách nội sinh. Nghiên cứu phân tích trên hai mẫu dữ liệu khác nhau. Mẫu thứ nhất bao gồm 9 quốc gia phát triển và mẫu thứ hai bao gồm tất cả các quốc gia phát triển và đang phát triển trong giai đoạn sau chiến tranh thế giới lần thứ hai. Trong nghiên cứu này, hai tác giả sử dụng mô hình "liên thế hệ" (overlappinggeneration) làm nền tảng lý thuyết cho việc phát triển khung phân tích và xác định mô hình hồi quy. Tác giả phân tích ảnh hưởng của bất bình đẳng thu nhập lên tăng trưởng kinh tế, trong đó có sự tác động đồng thời của các chính sách giảm thiểu bất bình đẳng lên cả hai yếu tố trên. Giả thuyết của mô hình "liên thế hệ" là nền kinh tế sẽ tiến đến trạng thái cân bằng của tăng trưởng hay trạng thái cân bằng của chính sách dựa trên giả định rằng các tương tác trong nền kinh tế xảy ra thông qua việc tối đa hóa mức độ hữu dụng của mỗi cá nhân, trong điều kiện các thế hệ tiếp nối về nguồn lực con người và chính sách là không giới hạn. Trong nghiên cứu đó, hai trạng thái cân bằng được tập trung vào là (i) cân bằng tăng trưởng, và (ii) cân bằng chính sách. Về phân tích cân bằng tăng trưởng, các tác giả giả định rằng độ hữu dụng của mỗi cá nhân trong chi tiêu gồm có hai thành phần: (i) chi tiêu hiện tại, và (ii) tiết kiệm hoặc tích lũy tài sản cho tương lai. Tối đa hóa hữu dụng trong chi tiêu sẽ dẫn đến trạng thái cân bằng của tăng trưởng kinh tế. Về phía cân bằng chính sách, Persson và Tabellini (1994) lập luận rằng phần tài sản 
tích lũy của mỗi cá nhân sẽ tạo nên sự khác biệt thu nhập trong xã hội ở tương lai; và việc tối đa hóa hữu dụng của mỗi cá nhân khi biểu quyết các chính sách làm thu hẹp khoảng cách trong thu nhập sẽ dẫn đến trạng thái cân bằng chính sách.

Dựa trên hai lập luận trên, yếu tố nội sinh được đưa vào trong mô hình phân tích. Do đó, tác giả sử dụng mô hình hồi quy bình phương nhỏ nhất hai giai đoạn để phân tích ảnh hưởng của bất bình đẳng thu nhập lên tăng trưởng kinh tế, trong đó yếu tố nội sinh là chính sách giảm thiểu bất bình đẳng. Nghiên cứu của Persson và Tabellini (1994) cung cấp bằng chứng thực nghiệm chỉ ra rằng bất bình đẳng trong thu nhập có tác động tiêu cực đến tăng trưởng kinh tế. Theo một cơ chế cụ thể, bất bình đẳng thu nhập trong một quốc gia sẽ tác động lên các chính sách nhằm giảm thiểu chúng, như thuế thu nhập cá nhân. Sau đó, các chính sách này sẽ tác động lên tăng trưởng kinh tế của quốc gia đó. Tuy nhiên, Persson và Tabellini (1994) cũng chỉ ra rằng cơ chế tác động trên chỉ xuất hiện mạnh mẽ ở các quốc gia dân chủ. Đối với các nước thuộc Tổ chức Hợp tác và Phát triển kinh tế (OECD), trong giai đoạn sau chiến tranh thế giới thứ 2 , bất bình đẳng thu nhập và chính sách giảm bất bình đẳng dường như không có mối liên hệ mạnh mẽ nào, đồng thời, tác động từ các chính sách đó lên tăng trưởng kinh tế cũng không có ý nghĩa thống kê.

Lin và cộng sản (2014) xem xét ảnh hưởng của bất bình đẳng lên tăng trưởng kinh tế giữa các nền kinh tế có mức độ phát triển khác nhau thông qua mô hình hồi quy ngưỡng với dữ liệu bảng. Các tác giả lập luận rằng nên xem xét mối quan hệ "bất bình đẳng - tăng trưởng kinh tế" trong phạm vi một quốc gia, giữa các tiểu bang hơn là phạm vi toàn cầu, giữa các quốc gia với nhau. Việc xem xét trong phạm vi một quốc gia mang lại nhiều lợi ích trong việc thống nhất đo lường bất bình đẳng, và cố định các điều kiện kiểm soát như chính sách và thể chế. Do đó, so sánh chéo giữa các tiểu bang có thể giảm thiểu độ lệch trong đo lường. Bước đầu, đánh giá ảnh hưởng của bất bình đẳng lên thu nhập được đánh giá theo góc nhìn tuyến tính và phương pháp hồi quy tuyến tính sử dụng "tác động cố định", nhằm cố định đặc tính của các bang, để phân tích mối quan hệ này. Tuy nhiên sau đó, các tác giả lập luận rằng có sự sụt giảm về bất bình đẳng khi thu nhập bình quân thực tăng lên. Kết quả hồi quy, khi đưa thêm "yếu tố tương tác" giữa bất bình đẳng và thu nhập bình quân thực vào mô hình tuyến tính, chỉ ra rằng ảnh hưởng từ bất bình đẳng lên tăng trưởng kinh tế chuyển từ tiêu cực sang tích cực khi tăng dần mức độ phát triển kinh tế.

Sau đó, Lin và cộng sự (2014) xây dựng mô hình phân tích dựa trên mối quan hệ phi tuyến tính giữa bất bình đẳng thu nhập và tăng trưởng kinh tế, và đề xuất sử dụng mô hình hồi quy ngưỡng với dữ liệu bảng (Hansen, 1999) nhằm xác định ngưỡng tác động của bất bình đẳng thu nhập lên tăng trưởng kinh tế. Từ kết quả thu được trên mô hình hồi quy ngưỡng với dữ liện bảng, Lin và cộng sự (2014) kết luận rằng trong điều kiện thu nhập thực bình quân ở mức thấp, dưới mức $12.400 \mathrm{USD} /$ năm, bất bình đẳng càng cao sẽ làm cho nền kinh tế tăng trưởng càng chậm. Tuy nhiên, khi thu nhập thực đạt mức độ cao, đạt từ $21.065 \mathrm{USD} /$ năm trở lên, thì tăng bất bình đẳng trong thu nhập sẽ làm tăng tốc độ tăng trưởng kinh tế. Do đó, các tác giả đề nghị các nhà hoạch định chính sách cần phải xem xét thận trọng mức độ thu nhập thực tế khi phát triển các chính sách phân phối lại thu nhập trong xã hội. 
Fawaz và cộng sự (2014) bóc tách mối quan hệ giữa bất bình đẳng trong thu nhập và tăng trưởng kinh tế ở hai nhóm nước đang phát triển, một là nhóm nước đang phát triển có thu nhập cao, hai là nhóm nước đang phát triển có thu nhập thấp. Phương pháp GMM được sử dụng để khai thác mô hình hồi quy động giữa bất bình đẳng thu nhập và tăng trưởng kinh tế. Trong mô hình hồi quy động, các biến đều được xem xét dưới dạng sai phân bậc nhất (first difference). Phương pháp GMM giúp giải quyết vấn đề nội sinh trong quan hệ của hai yếu tố được phân tích. Trong nghiên cứu của mình, Fawaz và cộng sự (2014) đưa thêm các yếu tố kiểm soát đặc trưng khác như vốn con người được đo lường bằng số năm đi học; và sự biến dạng của thị trường được đại diện bởi chỉ số cân bằng sức mua (PPP index). Kết quả từ nghiên cứu chỉ ra rằng, tồn tại mối quan hệ phi tuyến tính giữa bất bình đẳng thu nhập và tăng trưởng kinh tế. Kết luận chỉ ra rằng tác động của bất bình đẳng thu nhập lên tăng trưởng kinh tế ở hai nhóm nước đang phát triển là trái ngược với nhau. Đối với nhóm nước đang phát triển có thu nhập cao, bất bình đẳng trong thu nhập tương quan dương với tăng trưởng kinh tế, và ngược lại đối với các nước đang phát triển có thu nhập thấp.

Biswas và cộng sự (2017) nghiên cứu về vai trò của chính sách thuế trong việc giảm bất bình đẳng thu nhập và rồi tác động đến tăng trưởng kinh tế. Một khái niệm mới được giới thiệu, "nhân tố thu hẹp" (contraction factor), phản ánh phần thuế trung bình mà một cá nhân phải trả thêm cho mỗi một đồng tăng thêm trong thu nhập của họ, tính cho nhóm có thu nhập cao và thấp rồi so với mức thu nhập trung vị. Nhóm tác giả cũng đề cập đến vấn đề nội sinh trong mối quan hệ giữa "chính sách - bất bình đẳng thu nhập - tăng trưởng kinh tế", và đưa ra bộ hai nhóm biến công cụ. Nhóm biến công cụ thứ nhất là “cú sốc thuế ngoại sinh" (Mertens \& Ravn, 2013; Romer \& Romer, 2009, 2010). Nhóm biến công cụ thứ hai là nhóm biến về thể chế chính trị và nhân khẩu học. Biswas và cộng sự (2017) sử dụng cả hồi quy bình phương bé nhất (OLS), và hồi quy biến công cụ (IV) theo hướng tiếp cận GMM để phân tích cơ chế ảnh hưởng nói trên. Kết luận từ nghiên cứu này chỉ ra rằng chính sách thuế thực sự có tác động lên các hộ gia đình trên phương diện đầu tư (tiết kiệm), việc làm và chi tiêu. Tuy nhiên, chính sách thuế nhắm đến việc giảm khác biệt trong thu nhập giữa nhóm có thu nhập cao và nhóm trung vị trong nền kinh tế sẽ gây tác động tiêu cực lên tăng trưởng kinh tế quốc gia.

Barro (2000) lập luận rằng dưới những ngữ cảnh của mức độ không hoàn hảo của thị trường tín dụng, kinh tế chính trị, bất ổn xã hội, và tỷ lệ tiết kiệm, bất bình đẳng thu nhập có ảnh hưởng tới tăng trưởng kinh tế. Cụ thể hơn, sự không hoàn hảo của thị trường tín dụng thường được nhận biết thông qua thông tin bất cân xứng và những giới hạn của tổ chức pháp lý. Nếu thị trường vốn và tổ chức pháp lý có khuynh hướng thay đổi khi nền kinh tế phát triển, các ảnh hưởng gắn liền với mức độ không hoàn hảo của thị trường tín dụng trở nên rõ nét hơn ở các quốc gia nghèo so với các nước giàu. Chính vì thế, quy mô ảnh hưởng của bất bình đẳng thu nhập lên tăng trưởng kinh tế sẽ lớn hơn. Theo khía cạnh kinh tế chính trị, khi thu nhập trung bình vượt quá thu nhập trung vị, các chương trình và chính sách liên quan tới phân bổ lại nguồn thu nhập như thanh khoản chuyển nhượng, chi tiêu công và chính sách pháp lý dường như được ủng hộ nhiều bởi những lá phiếu chính trị nhằm xoa dịu thực trạng. Tác động của bất bình đẳng thu nhập tới tăng trưởng kinh tế cũng được nhìn nhận qua lăng kính bất ổn xã hội. Thật vậy, lăng kính cho rằng, người nghèo thường bị thúc đẩy vào con đường phạm tội, gây rối xã hội. Điều này nói lên rằng một phần nào đó nguồn lao động của quốc gia bị lãng phí, và kìm hãm 
tăng trưởng kinh tế. Theo quan điểm có liên quan đến tỷ lệ tiết kiệm, nhiều nhà kinh tế học đều thống nhất rằng tiết kiệm tư nhân tăng khi tổng thu nhập toàn nền kinh tế tăng. Nếu lập luận này đúng, các chính sách tái phân bổ thu nhập sẽ làm giảm tổng tiết kiệm của nền kinh tế.

Xuất phát từ mối quan hệ phi tuyến giữa bất bình đẳng thu nhập và tăng trưởng kinh tế được đề xuất bởi Kuznets (1955), Galor và Zeira (1993), Brueckner, Dabla-Norris, Gradstein, và Lederman (2018) chỉ ra cách thức tác động của bất bình đẳng thu nhập tác động tới tăng trưởng kinh tế trong các nước thuộc khối ASEAN. Thông qua lăng kính của sự không hoàn hảo của thị trường tín dụng, các tác giả nhấn mạnh rằng bất bình đẳng thu nhập càng lớn, đầu tư càng cao ở các quốc gia nghèo trong khi quan hệ nghịch biến giữa bất bình đẳng thu nhập và đầu tư được tìm thấy ở các nước giàu.

Risso, Punzo, và Carrera (2013) tìm thay mối liên hệ nghịch biến giữa tăng trưởng kinh tế và bất bình đẳng trong thu nhận ở Mê-xi-cô trong giai đoạn từ 1986 đến 2010. Lập luận của các tác giả dựa trên lý thuyết kinh tế học cổ điển cho rằng tăng trưởng phụ thuộc chủ yếu vào tỉ lệ tích trữ các nguồn lực sản xuất, và có liên hệ với tỉ lệ tiết kiệm. Theo đó, các vấn đề về phân phối thu nhập chỉ tác động đến tăng trưởng khi tỉ lệ tiết kiệm của các hộ gia đình biến đổi theo thu nhập hoặc của cải của họ. Bất bình đẳng có thể thúc đẩy tăng trưởng bởi tăng trưởng sản xuất cần có sự tích lũy vốn, ví dụ như những ngành sản xuất mới cần có một lượng vốn đầu tư lớn hơn. Như vậy, mức độ tập trung cao về thu nhập/tài sản sẽ tạo ra mức tích lũy vốn cao hơn, từ đó kích thích tăng trưởng thông qua sự phát triển của những ngành thâm dụng vốn.

Kennedy, Smyth, Valadkhani, và Chen (2017) tìm hiểu về tác động tăng trưởng của bất bình đẳng thu nhập ở Úc từ năm 1986 đến 2013, và cơ bản đưa ra kết quả là bất bình đẳng có tác động đến tăng trưởng kinh tế có độ trễ vài năm sau đó. Trong nghiên cứu đó, hai hướng tác động tích cực và tiêu cực của bất bình đẳng thu nhập đều được xem xét. Các tác giả cho thấy tác động tích cực của bất bình đẳng kinh tế thể hiện thông qua việc khuyến khích tiết kiệm và tạo ra nhiều động lực cho cá nhân làm việc cũng như đầu tư. Tương tự, tác động tiêu cực của bất bình đẳng thu nhập liên quan đến mức độ tập trung cao các nguồn lực kinh tế, và thị trường tín dụng không hoàn hảo.

\section{Dũ̃ liệu và phương pháp nghiên cứu}

\section{1. Đo luờng bất bình đẳng thu nhập}

Theo Haughton và Khandker (2009) bất bình đẳng về thu nhập được đo lường bằng 4 phương pháp phổ biến như:

(i) Tỉ lệ phân bổ $10 \%$ : đây là phương pháp đo lường đơn giản nhất. Chỉ số này là tỉ lệ về chi tiêu (hoặc thu nhập) trung bình của $10 \%$ dân số có thu nhập cao nhất so với chi tiêu (hoặc thu nhập) trung bình của $10 \%$ dân số có thu nhập thấp nhất. Chỉ số này còn có thể được áp dụng với các tỉ lệ phân phối khác nhau. Ví dụ như tính tỉ lệ thu nhập của $5 \%$ dân số giàu nhất trên $5 \%$ dân số nghèo nhất, hay tỷ lệ thu nhập của người dân ở phân vị thứ 95 so với thu nhập của người dân ở phân vị thứ 5 trên bảng phân phối thu nhập; 
(ii) Hệ số Gini về bất bình đẳng: đây là chỉ số được sử dụng rộng rãi nhất hiện nay trong các báo cáo hoặc nghiên cứu về bất bình đẳng thu nhập. Hệ số Gini bắt nguồn từ lý thuyết đường cong Lorenz về bất bình đẳng thu nhập của Lorenz (1906). Đường cong Lorenz mô tả phân phối của thu nhập thông qua hai trục giá trị: trục tung đo lường phần trăm cộng dồn thu nhập của toàn bộ dân số, trục hoành là phân phối cộng dồn của dân số hoặc của hộ gia đình từ nghèo nhất đến giàu nhất. Đường chéo $45 \%$ thể hiện bình đẳng hoàn hảo, nghĩa là không có sự phân biệt nào về thu nhập giữa các cá nhân hoặc giữa các hộ gia đình. Hệ số Gini được tính bằng tỉ số $\mathrm{A} /(\mathrm{A}+\mathrm{B})$. Khi $\mathrm{A}=0$, Gini $=0$, đường cong Lorenz trùng với đường chéo $45 \%$ thì hoàn toàn không có bất bình đẳng trong thu nhập. Khi $\mathrm{B}=0$, Gini $=1$, bất bình đẳng hoàn toàn xảy ra, thu nhập của toàn bộ xã hội sẽ tập trung vào một vài cá nhân giàu nhất xã hội.

(iii) Những đo lường entropy tổng quát: chỉ số Theil's L và Theil's T là hai chỉ số phổ biến trong đo lường entropy tổng quát $(\mathrm{GE})$ thường được dùng nhất. Công thức tính $\mathrm{GE}$ tổng quát như sau:

$$
G E(\alpha)=\frac{1}{\alpha(\alpha-1)}\left[\frac{1}{N} \sum_{i=1}^{N}\left(\frac{y_{i}}{\bar{y}}\right)^{\alpha}-1\right]
$$

Trong đó, $\bar{y}$ là thu nhập (hoặc chi tiêu) trung bình của cá nhân. Giá trị GE biến thiên từ không đến vô cùng, với giá trị GE bằng không thể hiện không có bất bình đẳng, và bất bình đằng càng cao khi giá trị GE càng lớn. Giá trị $\alpha$ trong phân loại GE thể hiện trọng số được cho bởi sự khác biệt về thu nhập khi được xem xét ở các phần khác nhau của phân phối thu nhập. Giá trị $\alpha$ càng bé thì giá trị tính toán GE sẽ càng tương thích với sự thay đổi ở bộ phận phân phối thấp nhất của thu nhập, ngược lại, giá trị $\alpha$ càng lớn thì GE càng nhạy cảm với sự thay đổi của các phân khúc phía trên trong phân phối thu nhập. Giá trị $\alpha$ được sử dụng phổ biến nhất là 0,1 , và 2 . GE(1) hay còn gọi là chỉ số Theil's $T$, được tính toán như sau:

$$
G E(1)=\frac{1}{N} \sum_{i=1}^{N} \frac{y_{i}}{\bar{y}} \ln \left(\frac{y_{i}}{\bar{y}}\right)
$$

Trong khi đó, $\mathrm{GE}(0)$ chính là chỉ số Theil’s L, được tính theo công thức:

$$
G E(0)=\frac{1}{N} \sum_{i=1}^{N} \ln \left(\frac{y_{i}}{\bar{y}}\right)
$$

(iv) Chỉ số bất bình đẳng Atkinson: Chỉ số này thể hiện phần trăm thu nhập mà một xã hội sẽ chi ra nhằm cải thiện sự ngang bằng về thu nhập của toàn bộ cư dân trong xã hội đó. Do đó, chỉ số này sẽ bị tác động bởi thái độ về bất bình đẳng của cả xã hội trên. Chỉ số Atkinson càng lớn thì thể hiện mức sẵn lòng trả của xã hội cho việc cải thiện bình đẳng về thu nhập càng cao, nghĩa là người dân sẵn sàng chấp nhận thu nhập thấp hơn để đánh đổi sự cân bằng trong phân phối thu nhập. Ngoài ra, chỉ số Atkinson còn có thể được phân ra để tính bất bình đẳng trong nội tại nhóm và giữa các nhóm cần xem xét. Vì sử dụng thái độ để đo lường bất bình đẳng, nên chỉ số này còn được dùng để đưa ra các đánh giá về phúc lợi xã hội nhằm hướng đến hoàn thiện các chính sách công phù hợp.

\subsection{Dũ liệu}

Nghiên cứu sử dụng dữ liệu, trải dài năm 2004 tới năm 2014 cho 63 Tỉnh/Thành Việt Nam, từ Niên giám thống kê và Bộ dữ liệu Điều tra mức sống hộ gia đình được thực hiện bởi Tổng cục Thống kê. Tuy nhiên, vì Bộ dữ liệu Điều tra mức sống hộ gia đình được công bố theo 
chu kỳ hai năm nên kết quả báo cáo được xây dựng từ các năm 2004, 2006, 2008, 2010, 2012 , 2014. Với nguồn dữ liệu từ Bộ dữ liệu Điều tra mức sống hộ gia đình, nghiên cứu sử dụng dữ liệu thu nhập cho nam giới và nữ giới để tính chỉ số bất bình đẳng thu nhập.

Với nguồn dữ liệu từ Niên giám thống kê, nghiên cứu sử dụng để thu thập dữ liệu về tổng sản phẩm trên địa phương theo giá hiện hành, vốn đầu tư thực hiện theo giá hiện hành, số lượng sinh viên cao đẳng, đại học đang theo học và tổng dân số của địa phương. Ngoài ra, chỉ số giá tiêu dùng trong từng năm cũng được thu thập để điều chỉnh lạm phát cho tổng sản phẩm trên địa phương và vốn đầu tư thực hiện với năm cơ sở là 2004. Trong trường hợp dữ liệu chỉ số giá tiêu dùng không có sẵn, để tối đa hóa số lượng quan sát có thể, nghiên cứu dùng chỉ số giá tiêu dùng của cả nước như là một phương án thay thế.

\section{Bảng 1}

Mô tả các biến chính sử dụng trong mô hình

\begin{tabular}{|c|c|c|c|c|c|}
\hline Biến & $\begin{array}{c}\text { Số lượng quan } \\
\text { sát }\end{array}$ & Trung bình & $\begin{array}{l}\text { Độ lệch } \\
\text { chuẩn }\end{array}$ & $\begin{array}{c}\text { Giá trị nhỏ } \\
\text { nhất }\end{array}$ & $\begin{array}{c}\text { Giá trị lớn } \\
\text { nhất }\end{array}$ \\
\hline $\ln$ (Income) & 324 & -4.660 & 1.261 & -6.991 & 2.185 \\
\hline \multicolumn{6}{|c|}{ Chì số Gini } \\
\hline $\ln$ (Inequality Male) & 378 & -1.095 & 0.148 & -1.457 & -0.732 \\
\hline $\begin{array}{l}\ln \text { (Inequality } \\
\text { FeMale) }\end{array}$ & 378 & -1.107 & 0.201 & -1.688 & -0.633 \\
\hline \multicolumn{6}{|c|}{ Chỉ số Theil } \\
\hline $\ln$ (Inequality Male) & 378 & -1.644 & 0.321 & -2.439 & -0.807 \\
\hline $\begin{array}{l}\ln \text { (Inequality } \\
\text { FeMale) }\end{array}$ & 378 & -1.685 & 0.399 & -2.890 & -0.703 \\
\hline
\end{tabular}

Nguồn: Tính toán của nhóm tác giả 


\section{Bảng 2}

Ma trận tương quan với chỉ số Gini được dùng để đo lường bất bình đẳng thu nhập

\begin{tabular}{|c|c|c|c|c|c|c|c|}
\hline & $\ln \left(\right.$ Income $\left._{i, t-1}\right)$ & $\ln \left(\right.$ Investment $\left.{ }_{i, t}\right)$ & $\ln \left(\right.$ Education $\left._{\mathrm{i}, \mathrm{t}}\right)$ & $\ln \left(\right.$ InequalityMale $\left.\mathrm{e}_{\mathrm{i}, \mathrm{t}}\right)$ & $\begin{array}{c}\ln \text { (InequalityM } \\
\text { ale }, \mathrm{t}-1)\end{array}$ & $\ln \left(\right.$ InequalityFemale $\left.e_{i, t}\right)$ & $\begin{array}{c}\text { In(InequalityFemale } e_{i, t-} \\
\text { 1) }\end{array}$ \\
\hline $\ln \left(\right.$ Income $\left._{i, t-1}\right)$ & 1.000 & & & & & & \\
\hline $\ln \left(\right.$ Investment $\left.\mathrm{i}_{\mathrm{i}, \mathrm{t}}\right)$ & -0.463 & 1.000 & & & & & \\
\hline $\ln \left(\right.$ Education $\left._{\mathrm{i}, \mathrm{t}}\right)$ & 0.531 & -0.068 & 1.000 & & & & \\
\hline $\ln \left(\right.$ InequalityMale $\left._{\mathrm{i}, \mathrm{t}}\right)$ & 0.001 & 0.018 & -0.180 & 1.000 & & & \\
\hline $\ln \left(\right.$ InequalityMale $\left._{\mathrm{i}, \mathrm{t}-1}\right)$ & -0.042 & -0.031 & -0.228 & 0.561 & 1.000 & & \\
\hline $\ln \left(\right.$ InequalityFemale $\left._{i, t}\right)$ & 0.094 & -0.154 & -0.010 & 0.461 & 0.366 & 1.000 & \\
\hline $\ln \left(\right.$ InequalityFemale $\left._{\mathrm{i}, \mathrm{t}-1}\right)$ & 0.099 & -0.125 & 0.0007 & 0.313 & 0.465 & 0.641 & 1.000 \\
\hline
\end{tabular}

Nguồn: Tính toán của nhóm tác giả

\section{Bảng 3}

Ma trận tương quan với chỉ số Theil được dùng để đo lường bất bình đẳng thu nhập

\begin{tabular}{|c|c|c|c|c|c|c|c|}
\hline & $\ln \left(\right.$ Income $\left._{\mathrm{i}, \mathrm{t}-1}\right)$ & $\ln \left(\right.$ Investment $\left._{\mathrm{i}, \mathrm{t}}\right)$ & $\ln \left(\right.$ Education $\left._{\mathrm{i}, \mathrm{t}}\right)$ & $\ln \left(\right.$ InequalityMale $\left._{\mathrm{i}, \mathrm{t}}\right)$ & $\ln \left(\right.$ InequalityMale $\left._{\mathrm{i}, \mathrm{t}-1}\right)$ & $\ln \left(\right.$ InequalityFemale $\left._{\mathrm{i}, \mathrm{t}}\right)$ & $\ln \left(\right.$ InequalityFemale $\left._{i, t-1}\right)$ \\
\hline $\ln \left(\right.$ Income $\left._{\mathrm{i}, \mathrm{t}-1}\right)$ & 1.000 & & & & & & \\
\hline $\ln \left(\right.$ Investment $\left._{\mathrm{i}, \mathrm{t}}\right)$ & -0.463 & 1.000 & & & & & \\
\hline $\ln \left(\right.$ Education $\left._{\mathrm{i}, \mathrm{t}}\right)$ & 0.531 & -0.068 & 1.000 & & & & \\
\hline $\ln \left(\right.$ InequalityMale $\left._{\mathrm{i}, \mathrm{t}}\right)$ & -0.0008 & 0.019 & -0.168 & 1.000 & & & \\
\hline $\ln \left(\right.$ InequalityMale $\left._{\mathrm{i}, \mathrm{t}-1}\right)$ & -0.052 & -0.025 & -0.212 & 0.565 & 1.000 & & \\
\hline $\ln \left(\right.$ InequalityFemale $\left._{\mathrm{i}, \mathrm{t}}\right)$ & 0.101 & -0.137 & -0.008 & 0.450 & 0.346 & 1.000 & \\
\hline $\ln \left(\right.$ InequalityFemale $\left._{\mathrm{i}, \mathrm{t}-1}\right)$ & 0.095 & -0.111 & -0.012 & 0.307 & 0.438 & 0.626 & 1.000 \\
\hline
\end{tabular}

Nguồn: Tính toán của nhóm tác giả 


\subsection{Phương pháp nghiên cúu}

Nghiên cứu kế thừa mô hình được đề cập trong nghiên cứu của Malinen (2013) để đánh giá và lượng hóa tác động của bất bình đẳng lên tăng trưởng kinh tế. Chính vì thế, mô hình ước lượng được diễn tả như sau:

$\ln \left(\right.$ income $\left._{\mathrm{i}, \mathrm{t}}\right)=\beta_{0}+\beta_{1} \ln \left(\right.$ income $\left._{\mathrm{i}, \mathrm{t}-1}\right)+\beta_{2} \ln \left(\right.$ investment $\left._{\mathrm{i}, \mathrm{t}}\right)+\beta_{3} \ln \left(\right.$ education $\left._{\mathrm{i}, \mathrm{t}}\right)+$ $\beta_{4} \ln \left(\right.$ inequalityMale $\left._{\mathrm{i}, \mathrm{t}}\right)+\beta_{5} \ln \left(\right.$ inequalityMale $\left._{\mathrm{i}, \mathrm{t}-1}\right)+\beta_{6} \ln \left(\right.$ inequalityFemale $\left._{\mathrm{i}, \mathrm{t}}\right)+$ $\beta_{7} \ln \left(\right.$ inequalityFemale $\left._{\mathrm{i}, \mathrm{t}-1}\right)+\mu_{\mathrm{i}, \mathrm{t}}$

Trong đó: Income $e_{i, t}$ là tổng thu nhập bình quân đầu người của địa phương i trong giai đoạn $\mathrm{t}$. Investmenti, là tổng vốn đầu tư thực hiện bình quân đầu người của địa phương i trong giai đoạn t. Education ${ }_{i, t}$ là tổng số lượng sinh viên cao đẳng, đại học đang theo học trên tổng dân số của địa phương $\mathrm{i}$ trong giai đoạn $\mathrm{t}$. InequalityMale $\mathrm{e}_{\mathrm{i}, \mathrm{t}}$ là chỉ số đo lường bất bình đẳng thu nhập giữa nam giới của địa phương $\mathrm{i}$ trong giai đoạn $\mathrm{t}$. InequalityFemale $\mathrm{i}_{\mathrm{i}, \mathrm{l}}$ là chỉ số đo lường bất bình đẳng thu nhập giữa nữ giới của địa phương $\mathrm{i}$ trong giai đoạn $\mathrm{t}$.

Về khía cạnh phương pháp hồi quy, nghiên cứu sử dụng phương pháp bình phương tối thiểu, phương pháp tác động cố định và phương pháp tác động ngẫu nhiên để đánh giá và lượng hóa sự tác động của bất bình đẳng thu nhập theo giới lên tăng trưởng kinh tế. Ba phương pháp ước lượng nêu trên cũng được áp dụng trong các nghiên cứu khác (Forbes, 2000; Lin et al., 2014; Malinen, 2013).

\section{Thảo luận kết quả}

Kết quả hồi quy cho tác động của bất bình đẳng thu nhập theo giới lên tăng trưởng kinh tế từ việc áp dụng ba phương pháp hồi quy - phương pháp bình phương tối thiểu, phương pháp tác động cố định và phương pháp tác động ngẫu nhiên - được trình bày trong Bảng 4 và Bảng 5.

\section{Bảng 4}

Kết quả hồi quy với chỉ số Gini được dùng để đo lường bất bình đẳng thu nhập

\begin{tabular}{|l|c|c|c|}
\hline & OLS & Fixed effect-OLS & $\begin{array}{c}\text { Random effect- } \\
\text { OLS }\end{array}$ \\
\hline Constant & $\begin{array}{c}-0.771 \\
(0.106)\end{array}$ & $\begin{array}{c}-2.530 * * * \\
(0.000)\end{array}$ & $\begin{array}{c}-0.875 \\
(0.201)\end{array}$ \\
\hline $\ln \left(\right.$ Income $_{\mathrm{i}, \mathrm{t}-1)}$ & $\begin{array}{c}0.767 * * * \\
(0.000)\end{array}$ & $\begin{array}{c}0.219 * \\
(0.097)\end{array}$ & $\begin{array}{c}0.743 * * * \\
(0.000)\end{array}$ \\
\hline $\ln \left(\right.$ Investment $\left._{\mathrm{i}, \mathrm{t}}\right)$ & $-0.224 *$ & $-0.782 * * *$ & -0.267 \\
& $(0.095)$ & $(0.000)$ & $(0.126)$ \\
\hline $\ln \left(\right.$ Education $\left._{\mathrm{i}, \mathrm{t}}\right)$ & $\begin{array}{c}0.105 \\
(0.184)\end{array}$ & $\begin{array}{c}0.207 * * \\
(0.033)\end{array}$ & $\begin{array}{c}0.112 \\
(0.189)\end{array}$ \\
\hline $\ln \left(\right.$ InequalityMale $\left._{\mathrm{i}, \mathrm{t}}\right)$ & $\begin{array}{c}0.446 \\
(0.120)\end{array}$ & $\begin{array}{c}0.681 * * * \\
(0.002)\end{array}$ & $\begin{array}{c}0.470 * \\
(0.059)\end{array}$ \\
\hline
\end{tabular}




\begin{tabular}{|c|c|c|c|}
\hline & OLS & Fixed effect-OLS & $\begin{array}{c}\text { Random effect- } \\
\text { OLS }\end{array}$ \\
\hline $\ln \left(\right.$ InequalityMale $\left._{\mathrm{i}, \mathrm{t}-1}\right)$ & $\begin{array}{c}0.125 \\
(0.686) \\
\end{array}$ & $\begin{array}{l}0.372 * \\
(0.076)\end{array}$ & $\begin{array}{c}0.127 \\
(0.647)\end{array}$ \\
\hline $\ln \left(\right.$ InequalityFemale $\left.{ }_{i, t}\right)$ & $\begin{array}{l}-0.476 \\
(0.103) \\
\end{array}$ & $\begin{array}{c}-0.584 * * * \\
(0.001)\end{array}$ & $\begin{array}{l}-0.491 \\
(0.100) \\
\end{array}$ \\
\hline $\begin{array}{l}\ln (\text { InequalityFemale } \\
\text { i,t- }\end{array}$ & $\begin{array}{l}-0.021 \\
(0.928)\end{array}$ & $\begin{array}{l}-0.070 \\
(0.722)\end{array}$ & $\begin{array}{l}-0.030 \\
(0.896)\end{array}$ \\
\hline Số lượng quan sát & 234 & 234 & 234 \\
\hline R-squared & 0.832 & 0.956 & 0.536 \\
\hline Hausman test & & $\begin{array}{l}608.17 \\
(0.000)\end{array}$ & \\
\hline LM test & & $\begin{array}{c}7.54 \\
(0.003)\end{array}$ & \\
\hline F test & & $\begin{array}{c}7.17 \\
(0.000)\end{array}$ & \\
\hline
\end{tabular}

Nguồn: Tính toán của nhóm tác giả. p-value ở trong dấu ngoặc đơn

\section{Bảng 5}

Kết quả hồi quy với chỉ số Thiel được dùng để đo lường bất bình đẳng thu nhập

\begin{tabular}{|c|c|c|c|}
\hline & OLS & Fixed effect-OLS & Random effect-OLS \\
\hline Constant & $\begin{array}{l}-0.823^{*} \\
(0.085)\end{array}$ & $\begin{array}{c}-2.906^{* * * *} \\
(0.000)\end{array}$ & $\begin{array}{l}-0.912 \\
(0.183)\end{array}$ \\
\hline $\ln \left(\right.$ Income $\left._{\mathrm{i}, \mathrm{t}-1}\right)$ & $\begin{array}{c}0.770 * * * \\
(0.000) \\
\end{array}$ & $\begin{array}{c}0.255^{* *} * \\
(0.027) \\
\end{array}$ & $\begin{array}{c}0.751 * * * \\
(0.000) \\
\end{array}$ \\
\hline $\ln \left(\right.$ Investment $\left._{i, t}\right)$ & $\begin{array}{l}-0.217 \\
(0.102)\end{array}$ & $\begin{array}{c}-0.759 * * * \\
(0.000)\end{array}$ & $\begin{array}{l}-0.255 \\
(0.142)\end{array}$ \\
\hline $\ln \left(\right.$ Education $\left._{\mathrm{i}, \mathrm{t}}\right)$ & $\begin{array}{c}0.102 \\
(0.192)\end{array}$ & $\begin{array}{l}0.199^{*} \\
(0.089)\end{array}$ & $\begin{array}{c}0.110 \\
(0.197)\end{array}$ \\
\hline $\ln \left(\right.$ InequalityMale $\left._{i, t}\right)$ & $\begin{array}{c}0.170 \\
(0.219)\end{array}$ & $\begin{array}{c}0.386^{* * * *} \\
(0.000)\end{array}$ & $\begin{array}{c}0.151 \\
(0.125)\end{array}$ \\
\hline $\ln \left(\right.$ InequalityMale $\left.\mathrm{i}_{\mathrm{i}, \mathrm{t}-1}\right)$ & $\begin{array}{c}0.076 \\
(0.590)\end{array}$ & $\begin{array}{c}0.108 \\
(0.527)\end{array}$ & $\begin{array}{c}0.075 \\
(0.536)\end{array}$ \\
\hline $\ln \left(\right.$ InequalityFemale $\left._{i, t}\right)$ & $\begin{array}{l}-0.222 \\
(0.143)\end{array}$ & $\begin{array}{c}-0.251^{* *} \\
(0.036)\end{array}$ & $\begin{array}{l}-0.228 \\
(0.131)\end{array}$ \\
\hline $\ln \left(\right.$ InequalityFemale $\left.e_{i, t-1}\right)$ & $\begin{array}{l}-0.011 \\
(0.929)\end{array}$ & $\begin{array}{l}-0.063 \\
(0.631)\end{array}$ & $\begin{array}{l}-0.013 \\
(0.912)\end{array}$ \\
\hline Số lượng quan sát & 234 & 234 & 234 \\
\hline R-squared & 0.542 & 0.685 & 0.530 \\
\hline
\end{tabular}




\begin{tabular}{|c|c|c|c|}
\hline & OLS & Fixed effect-OLS & Random effect-OLS \\
\hline Hausman test & & $\begin{array}{l}504.95 \\
(0.000)\end{array}$ & \\
\hline LM test & & $\begin{array}{c}7.27 \\
(0.003)\end{array}$ & \\
\hline F test & & $\begin{array}{c}3.68 \\
(0.000)\end{array}$ & \\
\hline
\end{tabular}

Nguồn: Tính toán của nhóm tác giả. p-vlue ở trong dấu ngoặc đơn

Kết quả hồi quy, khi cả hai chỉ số đo lường bất bình đẳng thu nhập - chỉ số Gini và chỉ số Theil - được sử dụng, chỉ ra rằng đầu tư tác động tiêu cực tới tăng trưởng kinh tế. Thật vậy, trong Bảng 4 , khi chỉ số Gini được sử dụng để đo lường bất bình đẳng thu nhập, hệ số hồi quy của đầu tư $-\ln ($ Investmenti,t) - lần lượt là $-0.224 ;-0.782$ và -0.267 đối với phương pháp bình phương tối thiểu, phương pháp tác động cố định và phương pháp tác động ngẫu nhiên, mặc dù hệ số không có ý nghĩa thống kê khi phương pháp tác động ngẫu nhiên được sử dụng. Bởi vì mô hình hồi quy có dạng tuyến tính logarit nên ý nghĩa hệ số hồi quy được giải thích như sau: đối với phương pháp bình phương tối thiểu, tính trung bình, nếu đầu tư tăng $1 \%$ thì tổng thu nhập bình quân đầu người giảm $0.224 \%$. Tương tự, liên quan tới phương pháp tác động cố định và phương pháp tác động ngẫu nhiên, tính trung bình, nếu đầu tư tăng $1 \%$ thì tổng thu nhập bình quân đầu người giảm $0.782 \%$ và $0.267 \%$. Tác động âm của đầu tư lên tăng trưởng kinh tế cũng được tái khẳng định trong Bảng 5 , nơi chỉ số Theil được sử dụng để đo lường bất bình đẳng thu nhập thay vì chỉ số Gini.

Liên quan tới tác động của giáo dục tới tăng trưởng kinh tế, cả ba phương pháp hồi quy ở trong Bảng 4 và Bảng 5 đều chỉ rõ tăng trưởng kinh tế hưởng lợi từ giáo dục vì hệ số hồi quy của biến giáo dục - $\ln ($ educationi,t) - lớn hơn không. Ví dụ, kết quả hồi quy trong Bảng 4 cho thấy hệ số hồi quy của biến số giáo dục lần lượt là $0.105 ; 0.207$ và 0.112 đối với phương pháp bình phương tối thiểu, phương pháp tác động cố định và phương pháp tác động ngẫu nhiên và chỉ có ý nghĩa thống kê cho phương pháp tác động cố định. Biến số giáo dục, được đặt dưới dạng $\log$, có hệ số được giải thích như sau: trong trường hợp phương pháp bình phương tối thiểu, tính trung bình, nếu tỷ lệ sinh viên tham gia giáo dục đại học, cao đẳng so với tổng dân số trên địa phương tăng $1 \%$ thì tổng thu nhập bình quân đầu người tăng $0.105 \%$. Liên quan tới hai phương pháp hồi quy còn lại, tính trung bình, nếu tỷ lệ sinh viên tham gia giáo dục đại học, cao đẳng so với tổng dân số trên địa phương tăng $1 \%$ thì tổng thu nhập bình quân đầu người tăng lần lượt $0.207 \%$ và $0.112 \%$. Kết quả hồi quy trong Bảng 5 cũng ủng hộ quan hệ dương ở trên, khi chỉ số Theil được sử dụng để đo lường bất bình đẳng thu nhập thay vì chỉ số Gini. Hơn thế nữa, phát hiện này cũng được tìm thấy trong các nghiên cứu trước đây (Malinen, 2013; Fawaz, Rahanama và Valcarcel, 2014; Forbes, 2000).

Đối với biến số chính trong nghiên cứu - bất bình đẳng thu nhập giữa nam giới và bất bình đẳng thu nhập giữa nữ giới - được đo lường qua chỉ số Gini và chỉ số Theil. Kết quả nghiên cứu chỉ ra rằng, đối với ba phương pháp hồi quy, bất bình đẳng giữa nam giới tác động dương tới tăng trưởng kinh tế trong khi bất bình đẳng giữa nữ giới tác động âm tới tăng trưởng kinh 
tế. Mối quan hệ dương này cũng được tìm thấy trong các nghiên cứu trước đây (Lin, Huang và Yeh, 2014; Fawaz, Rahanama và Valcarcel, 2014).

Trong Bảng 4, với mức ý nghĩa 10\%, hệ số hồi quy của biến đo lường bất bình đẳng thu nhập giữa nam giới - $\ln ($ InequalityMalei,t) - có ý nghĩa thống kê và có giá trị lần lượt là 0.681 và 0.470 cho phương pháp tác động cố định và phương pháp tác động ngẫu nhiên. Ý nghĩa của hệ số hồi quy được giải thích như sau: tính trung bình, nếu chỉ số Gini tăng $1 \%$ thì tổng thu nhập bình quân đầu người tăng $0.681 \%$ và $0.470 \%$. Tương tự, với mức ý nghĩa $1 \%$, hệ số hồi quy của biến đo lường bất bình đẳng thu nhập giữa nữ giới - $\ln ($ InequalityFemalei,t) - có ý nghĩa thống kê và có giá trị là -0.584 cho phương pháp tác động cố định. Có nghĩa là, tính trung bình, nếu chỉ số Gini tăng 1\% thì tổng thu nhập bình quân đầu người giảm $0.584 \%$.

Bên cạnh đó, khi chỉ số Theil được sử dụng (Bảng 5), với mức ý nghĩa 1\%, đối với phương pháp tác động cố định, hệ số hồi quy của biến đo lường bất bình đẳng thu nhập giữa nam giới có ý nghĩa thống kê và có quan hệ dương với tăng trưởng kinh tế. Trái ngược với quan hệ giữa bất bình đẳng thu nhập giữa nam giới và tăng trưởng kinh tế, một tác động âm của bất bình đẳng thu nhập giữa nữ giới và tăng trưởng kinh tế được tìm thấy.

\section{Kết luận và gọii ý chính sách}

\subsection{Kết luận}

Nghiên cứu này được thực hiện để đánh giá và lượng hóa tác động của bất bình đẳng thu nhập theo giới lên tăng trưởng kinh tế cho 63 Tỉnh/thành trong giai đoạn từ năm 2004 tới năm 2014. Bằng việc sử dụng Niên giám thống kê và Bộ dữ liệu Điều tra mức sống hộ gia đình, kết quả nghiên cứu chỉ ra rằng bất bình đẳng thu nhập giữa nam giới có tác động và có mối quan hệ dương với tăng trưởng kinh tế. Kết quả này không thay đổi khi 2 chỉ số đo lường bất bình đẳng thu nhập - chỉ số Gini và chỉ số Theil - được sử dụng. Ngoài ra, nghiên cứu còn chỉ ra rằng hệ số của bất bình đẳng thu nhập giữa nữ giới có tác động tới tăng trưởng kinh tế và có mối quan hệ âm, đầu tư có tác động tiêu cực tới tăng trưởng kinh tế và tăng trưởng kinh tế hưởng lợi từ giáo dục.

\subsection{Gọi ý chính sách}

Trong bối cảnh của Việt Nam trong giai đoạn vừa qua, vai trò quan trọng của nữ giới thường được xem là trụ cột của gia đình trên phương diện chăm lo công việc gia đình. Do vậy, thực tế chỉ ra rằng thu nhập của nữ giới trong hộ gia đình Việt Nam là rất hạn chế. Phần đông nữ giới Việt Nam vẫn chưa có cơ hội được tiếp cận công việc với mức thu nhập tương tự như nam giới, ngoại trừ nữ giới đang làm việc tại các thành phố lớn khi cơ hội làm việc là rất nhiều. Và do vậy, rất có thể đây là nguyên nhân dẫn đến kết luận rằng bất bình đẳng thu nhập của nữ giới tác động âm đến tăng trưởng kinh tế địa phương trong thời gian qua.

Bên cạnh đó, chênh lệch thu nhập của nam giới luôn đóng vai trò là trụ cột tài chính của gia đình. Sự chênh lệch thu nhập của nam giới góp phần tạo động lực và những nỗ lực cần thiết của những trụ cột gia đình trên phương diện tài chính. Những nỗ lực này có thể là nguyên nhân chính lý giải cho những đóng góp tích cực của chênh lệch thu nhập nam giới đến tăng trưởng kinh tế địa phương. 
Do vậy, các cơ quan quản lý nhà nước cần tiếp tục duy trì một môi trường cạnh tranh lành mạnh để nam giới có điều kiện thể hiện mình và đóp góp tích cực cho nền kinh tế địa phương. Các chính sách tái phân phối thu nhập cần được thực hiện hết sức thận trọng bởi vì điều này sẽ triệt tiêu nỗ lực của nam giới trong lao động, từ đó tác động tích cực đến tăng trưởng kinh tế địa phương. Trong dài hạn, phụ nữ nông thôn cần được giải phóng ra khỏi vai trò của người nội trợ toàn thời gian, hướng đến hình ảnh người phụ nữ hiện đại, mạnh mẽ và tham gia tích cực vào thị trường lao động nhằm mục đích đóng góp tích cực cho nền kinh tế địa phương.

\section{Tài liệu tham khảo}

Barro, R. J. (2000). Inequality and growth in a panel of countries. Journal of Economic Growth, 5(1), 5-32.

Biswas, S., Chakraborty, I., \& Hai, R. (2017). Income inequality, tax policy, and economic growth. The Economic Journal, 127(601), 688-727.

Brueckner, M., Dabla-Norris, E., Gradstein, M., \& Lederman, D. (2018). The rise of the middle class and economic growth in ASEAN. Journal of Asian Economics, 56, 48-58.

Fawaz, F., Rahnama, M., \& Valcarcel, V. J. (2014). A refinement of the relationship between economic growth and income inequality. Applied Economics, 46(27), 3351-3361.

Forbes, K. J. (2000). A reassessment of the relationship between inequality and growth. American Economic Review, 90(4), 869-887.

Galor, O., \& Zeira, J. (1993). Income distribution and macroeconomics. The Review of Economic Studies, 60(1), 35-52.

Goodwin, R. (1967). A growth cycle. In C. H. Feinstein (Ed.), Socialism, capitalism and economic growth. Cambridge, UK: Cambridge University Press.

Hansen, B. E. (1999). Threshold effects in non-dynamic panels: Estimation, testing, and inference. Journal of Econometrics, 93(2), 345-368.

Haughton, J., \& Khandker, S. R. (2009). Handbook on poverty + inequality. Washington, DC: World Bank Publications.

Kaldor, N. (1957). A model of economic growth. The Economic Journal, 67(268), 59-624.

Kennedy, T., Smyth, R., Valadkhani, A., \& Chen, G. (2017). Does income inequality hinder economic growth? New evidence using Australian taxation statistics. Economic Modelling, 65, 119-128.

Kuznets, S. (1955). Economic growth and income inequality. The American Economic Review, 45(1), 1-28.

Lewis, A. W. (1954). Economic development with unlimited supplies of labour. The Manchester School, 22(2), 139-191.

Lin, Y. C., Huang, H. C. R., \& Yeh, C. C. (2014). Inequality-growth nexus along the development process. Studies in Nonlinear Dynamics \& Econometrics, 18(3), 237-252. 
Malinen, T. (2013). Inequality and growth: Another look with a new measure and method. Journal of International Development, 25(1), 122-138.

McKinley, T. (2009). Revisiting the dynamics of growth, inequality and poverty reduction. Retrieved May 20, 2018, from http://citeseerx.ist.psu.edu/viewdoc/download?doi=10.1.1.472.9855\&rep=rep1\&type=p df

Mertens, K., \& Ravn, M. O. (2013). The dynamic effects of personal and corporate income tax changes in the united states. American Economic Review, 103(4), 1212-1247.

Oxfam. (2017). Thu hẹp khoảng cách cùng giảm bất bình đẳng ở Việt Nam [Closing the gap and reducing inequality in Vietnam]. Retrieved May 21, 2018, from https://www.oxfam.org/sites/www.oxfam.org/files/file_attachments/bp-vietnaminequality-120117-vn.pdf

Persson, T., \& Tabellini, G. (1994). Is inequality harmful for growth? The American Economic Review, 84(3599), 600-621.

Risso, W. A., Punzo, L. F., \& Carrera, E. J. S. (2013). Economic growth and income distribution in Mexico: A cointegration exercise. Economic Modelling, 35, 708-714.

Romer, C. D., \& Romer, D. H. (2009). A narrative analysis of postwar tax change. Retrieved May 22, 2018, from http://citeseerx.ist.psu.edu/viewdoc/summary?doi=10.1.1.370.4762

Romer, C. D., \& Romer, D. H. (2010). The macroeconomic effects of tax changes: Estimates based on a new measure of fiscal shocks. American Economic Review, 100(3), 763-801.

United Nations Development Programme. (2013). Humanity divided: Confronting inequality in developing countries. Retrieved Retrieved May 23, 2018 from http://www.undp.org/content/dam/ undp/library/ Poverty\%20Reduction/Inclusive\%20development/Humanity\%20Divided/HumanityDivi de d_Full-Report.pdf 